\title{
BENEFÍCIOS DOS PROBIÓTICOS PARA A MICROBIOTA INTESTINAL E SUA ADIÇÃO EM DERIVADOS LÁCTEOS E SUPLEMENTOS
}

\section{Benefits of probiotics for the gut microbiota and their addition in dairy products and supplements}

\author{
Tainara Luisa de Limal, Simone Weschenfelder ${ }^{l} *$
}

\begin{abstract}
RESUMO
Probióticos são micro-organismos vivos que conferem benefícios à saúde dos seres humanos, e seu consumo deve estar associado a uma alimentação equilibrada e a hábitos de vida saudáveis. O objetivo deste estudo foi verificar a diversidade de leites fermentados e suplementos com alegação de propriedade funcional por conter probióticos disponíveis em supermercados e farmácias da região do Vale do Sinos, Rio Grande do Sul, bem como apresentar os benefícios da ingestão de probióticos relacionados à microbiota intestinal. Foi realizada uma pesquisa nos supermercados avaliando-se rótulos de derivados lácteos (leites fermentados) que apresentavam probióticos. Para analisar a suplementação, foi realizada uma pesquisa nas farmácias da mesma região, verificando-se os suplementos com probióticos, as cepas e quantidades de micro-organismos presentes. Foram identificados seis tipos de leites fermentados e seis tipos de suplementos. Os benefícios dos probióticos sobre a microbiota humana incluem fatores como efeitos antagônicos, competição e efeitos imunológicos, aumentando a resistência a micro-organismos patogênicos. Apesar de existirem inúmeros estudos sinalizando os benefícios dos probióticos para a saúde, ainda há uma variedade muito pequena de alimentos e suplementos que os contenham e que estejam disponíveis no mercado.
\end{abstract}

Palavras-chave: micro-organismos benéficos; alimento funcional; flora intestinal.

1 Universidade FEEVALE, ERS-239, 2.755, 93525-075, Novo Hamburgo, RS, Brasil. E-mail: simone.weschenfelder@yahoo.com.br

* Autor para correspondência 


\begin{abstract}
Probiotics are living microorganisms that confer health benefits to humans, and their consumption must be associated with a balanced diet and healthy lifestyle. The aim of this study was to verify the diversity of fermented milks and supplements with the claim of functional property by "probiotic product" allegation available in supermarkets and pharmacies in the Vale do Sinos region, Rio Grande do Sul, as well as to present the benefits of ingestion of probiotics related to intestinal microbiota. A research was conducted in supermarkets evaluating labels of dairy products (fermented milks) in which it was written that the product contained probiotics. To analyze the supplements' labels, a survey was conducted at pharmacies in the same region, checking for supplements with probiotics, strains and amounts of microorganisms present. Six types of fermented milks and six types of supplements were identified. The benefits of probiotics on human microbiota include factors such as antagonistic effects, competition and immunological effects, therefore increasing the body resistance to pathogenic microorganisms. Although there are numerous studies signaling the health benefits of probiotics, there is still a very small variety of foods and supplements containing them that are available to be bought.
\end{abstract}

Keywords: beneficial microorganisms; functional food; intestinal flora.

\section{INTRODUÇÃO}

Segundo o Guia Alimentar para a População Brasileira (BRASIL, 2014), na maioria dos países economicamente emergentes, como o Brasil, os casos de diabetes mellitus tipo II e obesidade têm aumentado rapidamente. Do mesmo modo, evoluem outras doenças crônicas relacionadas ao alto consumo de calorias, como hipertensão arterial sistêmica, cardiopatias e certos tipos de câncer (BUJALANCE et al., 2007; SAAD et al., 2011; COOK et al., 2012).

Diante dessa realidade, a busca pela qualidade de vida vem sendo cada vez mais comum, principalmente quando o assunto é alimentação. A indústria alimentícia tem se visto desafiada pelos consumidores, que procuram produtos que sejam saborosos, visualmente atrativos e que, ao mesmo tempo, visam à saúde e ao bem estar (SAAD et al., 2011).

Entre esses alimentos, encontramos os alimentos funcionais. A classificação dos alimentos funcionais pode ser de acordo com o alimento em si ou conforme os componentes bioativos nele presentes, como os probióticos, as fibras, os fitoquímicos, as vitaminas e os minerais essenciais, além de determinados peptídeos e proteínas (SAAD et al., 2011).

De acordo com a Food and Agriculture Organization of United Nations (2001), probióticos são micro-organismos vivos, que, quando administrados em quantidades adequadas, conferem benefícios à saúde do hospedeiro. Entre os probióticos mais estudados e empregados como ingredientes funcionais, destacam-se os lactobacilos e as bifidobactérias.

O benefício dos probióticos sobre a microbiota humana inclui fatores como efeitos antagônicos, competição e efeitos imunológicos, aumentando a resistência contra micro-organismos patogênicos. A utilização de culturas bacterianas probióticas estimula a multiplicação de bactérias benéficas, impedindo a proliferação de bactérias potencialmente prejudiciais, reforçando os mecanismos de defesa naturais do hospedeiro (COOK et al., 2012). Além de auxiliar 
na manutenção da saúde, os probióticos impedem a reabsorção de compostos aminados indesejáveis, decompondo ácidos biliares, biodisponibilizando minerais (como cálcio e ferro), diminuindo a incidência de doenças coronárias e ajudando na digestão. Ressalta-se, ainda, que essa microbiota estimula o sistema imunológico e atividades antitumorogênicas e antimutagênicas (BUJALANCE et al., 2007; COOK et al., 2012).

Já a fisiologia do intestino, sua composição e a atividade do ecossistema microbiano responsável pela sua colonização, têm interessado para grande parte dos processos de desenvolvimento de alimentos funcionais, o que pode ser confirmado pelos atuais produtos na área de probióticos, prebióticos e simbióticos. A possibilidade de optar por benefícios adicionais à saúde tem atraído os consumidores a escolherem produtos funcionais em troca dos tradicionais. Entretanto, esse benefício não pode ser facilmente percebido pelas características físicas dos produtos ou pelo odor e textura, o que leva o consumidor a acreditar nas informações citadas no rótulo (ARES et al., 2009).

No Brasil, a Comissão Técnicocientífica de Assessoramento em Alimentos Funcionais e Novos Alimentos, criada junto à Câmara Técnica de Alimentos, tem avaliado os produtos com alegações de propriedades funcionais de saúde aprovados no país. No que se refere aos probióticos, desde 2016 as indústrias precisam apresentar a caracterização do micro-organismo empregado, o perfil de resistência a antimicrobianos e informações sobre a base genética da resistência antimicrobiana, a determinação da atividade hemolítica para espécies com potencial hemolítico, estudos disponíveis na literatura que descrevam efeitos adversos observados com a cepa em questão, além da demonstração de eficácia e da viabilidade ao longo do prazo de validade (BRASIL, 2018).
Para a microbiota intestinal estar balanceada não é necessária a ingestão de microorganismos probióticos, mas a administração destes traz um impacto benéfico para saúde do consumidor (TANNOCK, 1998). Para garantir tal benefício, deve-se manter uma seleção adequada de cepas para o processamento de produtos probióticos, principalmente os derivados lácteos (MATTILA-SANDHOLM et al., 2002, VINDEROLA; REINHEIMER, 2000), uma vez que a sobrevivência das bactérias probióticas no produto alimentício é fundamental (SAAD et al., 2011; BRASIL, 2018).

Os probióticos podem ser ingeridos via alimentos ou suplementos alimentares que segundo o marco regulatório da ANVISA, são todos os produtos de ingestão oral, apresentados em formas farmacêuticas, destinados a suplementar a alimentação de indivíduos saudáveis com nutrientes, substâncias bioativas, enzimas ou probióticos, isolados ou combinados. A concentração e a variedade de probióticos nesses produtos são distintas e existem poucas pesquisas de referência no Brasil, justificando-se, assim a relevância deste estudo. Assim, o objetivo do trabalho foi verificar a diversidade de derivados lácteos e suplementos alimentares comercializados em supermercados e farmácias da região do Vale do Sinos (RS) com alegação de probióticos, apresentando os principais benefícios da ingestão de probióticos relacionados à microbiota intestinal.

\section{MATERIAL E MÉTODOS}

\section{Diversidade de alimentos e suplementos com alegação de probióticos}

Para verificar a diversidade de alimentos com alegação de propriedade funcional por conter probióticos foi realizada uma pesquisa em um supermercado de um município de 
aproximadamente 200.000 habitantes da região do Vale dos Sinos, Rio Grande do Sul, avaliando-se rótulos de derivados lácteos (leites fermentados) que apresentavam a alegação de probióticos no rótulo, tabulando a denominação de venda do alimento, marca e se havia a indicação da cepa probiótica empregada.

Em relação à suplementação, foram analisadas as variedades de suplementos alimentares com alegação de probióticos comercializados em 4 farmácias do mesmo município onde foram coletados os dados dos leites fermentados, e quais as cepas de microorganismos eram utilizadas, como também as quantidades de probióticos presentes, expressos em UFC.

\section{Benefícios dos probióticos relacionados à microbiota intestinal}

Para verificar os benefícios dos probióticos relacionados à microbiota intestinal, utilizaram-se, para compor o corpus de referência, artigos científicos que abordavam aspectos relativos aos probióticos. Os critérios de inclusão para seleção dos artigos foram: somente artigos publicados e distribuídos entre pesquisa e revisão disponíveis na Scientific Eletronic Library Online (Scielo). As palavras-chave utilizadas foram: probióticos, alimentos funcionais e microbiota intestinal, que apareceram com ênfase nos trabalhos que relatavam benefícios à microbiota intestinal, publicados em inglês e português.

\section{RESULTADOS E DISCUSSÃO}

Para o alimento ter a eficácia de um probiótico, as bactérias devem estar ativas até o fim do prazo de validade do produto, e armazenamento refrigerado, é fundamental (SAAD, 2006). Vale destacar que, na literatura, existem relatos de mais microorganismos com potencial probiótico já estudados além daqueles que são encontrados nos produtos de supermercados e farmácias. Os alimentos e suplementos encontrados com alegação de probióticos estão apresentados nas Tabelas 1 e 2 .

Foram identificados seis tipos de leites fermentados probióticos no supermercado pesquisado com valores variando de $\mathrm{R} \$ 0,99$ a $\mathrm{R} \$ 5,99$ a unidade. Ao comparar o presente levantamento com o de Melo et al. (2013), que encontrou quarenta e quatro produtos nos supermercados do Rio de Janeiro e Grande Rio, observa-se que a diferença na quantidade de produtos encontrados foi considerável. O mercado brasileiro possui uma considerável variedade de marcas e tipos de produtos alimentícios por ser um país com expressiva produção de matéria prima alimentar, possibilitando uma série de combinações entre diferentes tipos de frutas e cereais. Foi possível observar no estudo de Melo et al. (2013) um maior número de produtos probióticos no grupo leite e laticínios com $100 \%$ dos produtos contendo proteínas lácteas na lista de ingredientes. Ao que tudo indica, a diferença entre o número de produtos contendo alegação de probióticos no rótulo do estudo de Melo et al. (2013) e o presente estudo se deve ao tamanho e desenvolvimento da região pesquisada.

No Brasil, dos diversos leites fermentados disponíveis no mercado, apenas alguns contêm no rótulo a identificação do micro-organismo presente (KEMPKA et al., 2008). Não havia declaração de número de UFC dos probióticos em nenhum dos rótulos avaliados dos derivados lácteos do presente estudo. Nas farmácias foram encontrados seis tipos de suplementos, com valores variando de $\mathrm{R} \$ 49,90$ a $\mathrm{R} \$ 129,90$ a caixa e constam as cepas empregadas e número de UFC utilizadas no rótulo dos mesmos. $\mathrm{O}$ acesso à informação ampla e abrangente nos rótulos aprimora as escolhas alimentares e eleva o nível de segurança alimentar e nutricional da população como um todo. 
Tabela 1 - Diversidade de alimentos com alegação de probióticos encontrados em supermercados da região do Vale do Sinos

\begin{tabular}{lll}
\hline $\begin{array}{l}\text { Denominação de venda } \\
\text { do produto }\end{array}$ & Marca & Cepa(s) empregada(s) \\
\hline Leite fermentado & Yakult & Lactobacillus casei shirota \\
\hline Leite fermentado Chamyto & Nestlé & Lactobacillus vivos paracasei \\
\hline Leite Fermentado Actimel & Danone & Lactobacillus defensis \\
\hline $\begin{array}{l}\text { Leite Fermentado Activia } \\
\text { zero lactose }\end{array}$ & Danone & Bifidobacterium animalis DN173 \\
\hline $\begin{array}{l}\text { Iogurte Grego com polpa } \\
\text { de banana e chia }\end{array}$ & Yorgus & $\begin{array}{l}\text { S. termophilus, } \text { Lulgaricus, } \\
\text { L. acidophilus, Bifidus, L.casei }\end{array}$ \\
\hline $\begin{array}{l}\text { Iogurte Integral com } \\
\text { preparado de mirtilos }\end{array}$ & Piá & $\begin{array}{l}\text { Lactobacillus acidophilus } \mathrm{e} \\
\text { Bifidobacterium lactis }\end{array}$ \\
\hline
\end{tabular}

Tabela 2 - Diversidade de suplementos com alegação de probióticos encontrados em farmácias da região do Vale dos Sinos

\begin{tabular}{llc}
\hline Suplemento & Cepas & UFC \\
\hline \multirow{3}{*}{ Probiatop } & L. acidophillus SD 5221 & $10^{9}$ \\
& L. rhamnosus SD5675 & $10^{9}$ \\
& L. paracasei SD 5275 & $10^{9}$ \\
& B. lactis SD5674 & $10^{9}$ \\
\hline Simbioflora & L. acidophillus & $10^{9}$ \\
& L. rhamnosus & $10^{9}$ \\
& L. paracasei & $10^{9}$ \\
& B. lactis & $10^{9}$ \\
ProVance & Frutooligossacarídeo (FOS) & $5,5 \mathrm{~g}$ \\
\hline Bidrilac & Lactobacillus reuteri & $10^{8}$ \\
\hline ProLive & L. acidophillus & $10^{9}$ \\
& B. lactis & $10^{9}$ \\
\hline \multirow{3}{*}{ L. acidophillus } & $10^{9}$ \\
\hline & L. acidophilus & $10^{9}$ \\
& L. casei & $10^{9}$ \\
& B. lactis & $10^{9}$ \\
& L. plantarum & $10^{9}$ \\
& L. salivarius & $10^{9}$ \\
& B. bifidum & $10^{9}$ \\
& B. longum & $10^{9}$ \\
& L. rhamnosus & $10^{9}$ \\
\hline & & \\
& &
\end{tabular}


Em relação aos benefícios dos probióticos relacionados à microbiota intestinal, na pesquisa realizada por Costa et al. (2012), foram avaliadas in vitro duas linhagens de lactobacilos e uma de bifidobactéria quanto ao potencial de inibição de micro-organismos patogênicos relacionados nas mais comuns toxinfecções alimentares, em que também podem ocorrer diarreias e desconfortos gástricos. As espécies Lactobacillus acidophilus, Lactobacillus plantarum e Bifidobacterium lactis apresentaram inibição contra Staphylococcus aureus, Bacilus cereus, Escherichia coli e Salmonella enteritidis. Segundo o autor, o impedimento dos agentes patogênicos ocorreu devido à produção de ácido lático e acético, produzidos a partir da fermentação. A produção destes ácidos potencializa o efeito antimicrobiano e, consequentemente, resulta no equilíbrio da microbiota intestinal.

Diversos fatores externos podem interferir na microbiota normal do nosso intestino, como a dieta, o uso de antibióticos, o estresse, o fumo, os tratamentos quimioterápicos e radioterápicos, além do envelhecimento (SANTOS et al., 2008).

Uma microbiota intestinal equilibrada é reflexo do desempenho normal das funções fisiológicas do hospedeiro, o que irá garantir a qualidade de vida do próprio. A microbiota intestinal exerce um importante papel em vários processos bioquímicos no hospedeiro, assim, quando ela se encontra em equilíbrio, impede que micro-organismos patógenos realizem suas funções. Quando ela se encontra em desequilíbrio, pode resultar na proliferação desses patógenos e provocar infecções bacterianas (SAAD, 2006).

Além dos aspectos relacionados ao processamento, a regulamentação e o monitoramento dos produtos com alegação funcional através das bactérias probióticas é um desafio para várias agências de segurança alimentar por todo o mundo, pois consiste em avaliar constantemente a relação entre a alegação funcional que aumenta o valor agregado do produto com a real viabilidade e eficácia desses micro-organismos para a saúde humana. Importante ressaltar que os benefícios são alcançados quando a ingestão dos micro-organismos é acompanhada de uma dieta equilibrada juntamente com hábitos de vida saudáveis.

Os prováveis mecanismos de ação dos probióticos são ativados pela competição por sítios de adesão, o que causa a formação de uma barreira física contra agentes patogênicos (LAZADO et al., 2011). Além disso, estimula a produção de substâncias antibacterianas, que tem ação bacteriostática ou bactericida em relação às bactérias patogênicas (LIMA et al., 2007). Além da competição por nutrientes, que impede a colonização de outros microorganismos, a ação dos probióticos causa a inativação das toxinas e seus receptores e a estimulação da fagocitose e das respostas imunológicas específicas e inespecíficas contra agentes patogênicos (SILVA et al., 2004; MATSUMOTO et al., 2005).

Segundo Stefe et al. (2008), muitos efeitos probióticos são mediados pela regulação imune, principalmente pelo controle do balanço das citocinas, pró e antiinflamatórias, o que melhora as respostas de imunoglobulina A (IgA).

A microbiota do intestino é constituída por um aglomerado de micro-organismos ao longo de todo trato gastrintestinal; esse aglomerado constituinte é variável e está situado entre a mucosa e o lúmen intestinal (BEDANI; ROSSI, 2009). É formada por aproximadamente 1.014 micro-organismos e localiza-se no cólon, constituindo-se, preferencialmente, por bactérias anaeróbicas estritas. Os micro-organismos que compõem a microbiota pertencem a mais de mil espécies de bactérias diferentes, que, por simbiose, mantêm uma relação (tais como o comensalismo) ou exercem papel patogênico 
com o hospedeiro (WILLIAMS, 2010).

Em casos de desequilíbrio na microbiota intestinal, podem surgir alguns distúrbios como diarreias, constipação, doenças inflamatórias intestinais e câncer de cólon (BADARÓ et al., 2009).

A diarreia consiste na eliminação anormal de fezes que podem ser líquidas ou moles em grandes quantidades. Os principais problemas são a eliminação de água e a má absorção de micro e macro nutrientes que pode levar à desnutrição. As diarreias podem ser de causa infecciosa, alimentar ou pelo uso de medicamentos. Em relação a essa disfuncionalidade, muitas vezes o uso de probióticos pode ser de extrema importância, quando utilizados de forma preventiva e terapêutica (PROTIC et al., 2005; SAZAWAL et al., 2006).

Já a disbiose, como é denominado o desequilíbrio da microbiota, pode manifestarse pela qualidade da alimentação ingerida (se é rica em gorduras saturadas e pobre em fibras, por exemplo), pelo estilo de vida (o que inclui o modo sedentarismo e o envelhecimento), por desordens intestinais frequentes, por câncer, por cirurgias, por doenças hepáticas e renais, por anemias, por estresse, por fatores imunológicos, por terapia com antibióticos, entre outros (ANTUNES et al., 2007). Para evitar a disbiose, os probióticos podem ser um importante aliado, uma vez que auxiliam na manutenção da microbiota.

Outro benefício dos probióticos em relação ao trato gastrointestinal é o auxílio na redução das manifestações sintomáticas das cólicas abdominais, relacionadas com a motilidade anormal e a síndrome de intestino irritável, visto que os probióticos interferem na regulação dos receptores opióides e canabióides das células epiteliais intestinais regulando a dor visceral (CAMILLERI, 2006).

\section{CONCLUSÃO}

A ingestão de probióticos apresenta inúmeros benefícios à microbiota intestinal. Contudo, apesar de inúmeros estudos sinalizando os benefícios ainda existe pouca diversidade de alimentos e suplementos disponíveis no mercado da região do Vale do Sinos (RS). Além disso, no caso dos suplementos, o custo é consideravelmente elevado, dificultando o acesso principalmente à população de baixa renda.

Cabe aos profissionais da saúde, primeiramente, apropriar-se dessa temática tão relevante, e, posteriormente, instruir e sugerir à população os probióticos, salientando e explanando sua importância em relação à manutenção da saúde, principalmente no que se refere à microbiota intestinal.

\section{REFERÊNCIAS}

ANTUNES, A. E. C. et al. Probióticos: microorganismos funcionais. Nutrire, v. 3, n. 32, p. 103-122, 2007.

ARES, G.; GIMÉNEZ, A.; GÁMBARO, A. Consumer perceived healthiness and willingness to try functional milk desserts: influence of ingredient name and health claim. Food Quality and Preference, v. 20, n. 1, p. 50-56, 2009. DOI:10.1016/j. foodqual.2008.07.002

BADARÓ, A. C. L et al. Alimentos probióticos: aplicações como promotores da saúde humana. Revista Digital de Nutrição, v. 3, n. 4, p. 396-410, 2009.

BEDANI, R; ROSSI, E. A. Microbiota intestinal e probióticos: implicações sobre câncer de cólon. Jornal Português de Gastrenterologia, v. 16, n. 1, p. 19-28, 2009.

BRASIL. MINISTÉRIO DA SAÚDE. AGÊNCIA NACIONAL DE VIGILÂNCIA SANITÁRIA (ANVISA). Resolução da 
Diretoria Colegiada - RDC n ${ }^{\circ} 241$, de 26 de julho de 2018. Dispõe sobre os requisitos para comprovação da segurança e dos benefícios à saúde dos probióticos para uso em alimentos. Diário Oficial da União, Brasília, 27 jul. 2018. Seção I, p. 97.

BRASIL. MINISTÉRIO DA SAÚDE. SECRETARIA DE ATENÇÃO À SAÚDE. Guia alimentar para a população brasileira. 2. ed., 1. reimpr., 2014. 156 p.

BUJALANCE, C. et al. A probiotic strain of Lactobacillus plantarum stimulates lymphocyte responses in immunologically intact and immunocompromised mice. International Journal of Food Microbiology, v. 113, n. 1, p.28-34, 2007. DOI: 10.1016/ j.ijfoodmicro.2006.07.014

CAMILLERI, M. Probiotics and irritable bowel syndrome: rationale, putative mechanisms, and evidence of clinical efficacy. Journal of Clinical Gastroenterology, v. 40, n.3, p. 264- 269, 2006.

COOK, M. T. et al. Microencapsulation of probiotics for gastrointestinal delivery. Journal of Controlled Release, v. 162, n. 1, p.56-67, 2012. DOI: $10.1016 / \mathrm{j}$. jconrel.2012.06.003

COSTA, G. N. et al. Atividade antimicrobiana de Lactobacillus e Bifidobacterium frente a microrganismos patogênicos "in vitro". SEMINA Ciências Agrárias, v. 33, n. 5, p. 1839-1846, 2012.

FOOD AND AGRICULTURE ORGANIZATION OF THE UNITED NATIONS, WORLD HEALTH ORGANIZATION. Evaluation of health and nutritional properties of probiotics in food including powder milk with live lactic acid bacteria. Córdoba, 2001. 34p. Disponível em: ftp://ftp.fao.org/es/esn/ food/probioreport_en.pdf Acesso em: 29 abr. 2018.

KEMPKA, A. P. et al. Formulação de bebida láctea fermentada sabor pêssego utilizando substratos alternativos e cultura probiótica. Ciência e Tecnologia de Alimentos, v. 28(Supl.), p. 170-177, 2008.

LAZADO, C. C. et al. In vitro adherence of two candidate probiotics from Atlantic cod and their interference with the adhesion of two pathogenic bacteria. Veterinary Microbiology, v. 148, n. 2-4, p. 252-259, 2011.

LIMA. E. T. et al. Evaluation in vitro of the antagonistic substances produced by Lactobacillus spp. isolated from chickens. Canadian Journal of Veterinary Research, v. 71, n. 2, p. 103-107, 2007.

MATSUMOTO, S. et al. Probiotic Lactobacillus-induced improvement in murine chronic inflammatory bowel disease is associated with the down-regulation of proinflammatory cytokines in lamina propria monoclear cell. Clinical and Experimental Immunology, v. 140, n. 3, p. 417-429, 2005.

MATTILA-SANDHOLM et al. Technological challenges for future probiotic foods. International Dairy Journal, v. 12, n. 2-3, p. 173-182, 2002.

MELO, T. A. et al. Levantamento e caracterização dos produtos probióticos disponíveis no mercado varejista da região metropolitana do Rio de Janeiro. Revista Rede de Cuidados em Saúde, v. 13, n. 1, p.1-13, nov. 2013.

PROTIC, M. et al. Mechanism of diarrhea in microscopic colitis. World Journal of 
Gastroenterology, v. 11, n. 35, p. 5535-5539, 2005.

RENHE, I. R. et al. Prebióticos e os benefícios de seu consumo na saúde. Revista Brasileira de Nutrição, v. 23, n. 2, p. 120124, 2008.

SAAD, S. M. I.; CRUZ, A. G.; FARIA, J. A. F. Probióticos e prebióticos em alimentos: fundamentos e aplicações tecnológicas. São Paulo: Livraria Varela, 2011. 672 p.

SAAD, S. M. I. Probióticos e prebióticos: o estado da arte. Brazilian Journal of Pharmaceutical Sciences, v. 42, n. 1, p.1-16, mar. 2006.

SANTOS, F. L. et al. Utilização de probióticos na redução da anemia ferropriva. Diálogos e Ciência, v. 7, n. 4, p. 13-18, 2008.

SAZAWAL, S. et al. Efficacy of probiotics in prevention of acute diarrhoea: a meta-analysis of masked, randomized, placebo-controlled trials. The Lancet Infectious Diseases, v. 6, n. 6, p. 374-382, 2006.
SILVA, A. M. et al. Effect of Bifidobacterium longum ingestion on experimental salmonellosis in mice. Journal of Applied Microbiology, v. 97, n. 1, p. 29-37, 2004.

STEFE, C. A. et al. Prebióticos, probióticos e simbióticos. Saúde e Ambiente, v. 1, n. 3, p.16-33, jan. 2008.

TANNOCK, G. W. Studies of the intestinal microflora: a prerequisite for the development of probiotics. International Dairy Journal, v. 8 , n. 5-6, p. 527-533,1998.

VINDEROLA, C. G.; REINHEIMER, J. A. Enumeration of Lactobacillus casei in the presence of L. acidophilus, bifidobacteria and lactic starter bacteria in fermented dairy products. International Dairy Journal, v. 10, n. 4, p. 271-275, 2000.

WILliams, N. T. Probiotics. American Journal of Health-System Pharmacy, v. 67, n. 6, p. 449-458, 2010. DOI: 10.2146/ ajhp090168. 